which has only the solutions $\dot{\theta}=$ constant, or $\theta=0$. It is easy to deduce from his equations, e.g.

$$
\frac{\Theta}{m g a}=\frac{R}{g}
$$

(Schuler's eq. 5)

and

$$
\frac{J}{m g a \cdot u \cdot \cos \varphi}=\frac{R}{g}
$$

(Schuler's par. $3^{1}$ )

by simply multiplying both members by $g$, that his apparatus is independent of $g$ !一 and hence, again, fundamentally different from that characterized by equations (1) and (2), above. In inertial navigation, it is Earth that is in tune, and there is no possibility of altering the period by tinkering with the device. I do not think that anyone can produce an inertial navigator with any other period, as, e.g., the period of 'about thirty minutes' reported by Schuler in his 1923 paper, par. 31 , as his best approach to an apparatus 'with full 84 -minute period.'

\title{
The Schuler Pendulum and Inertial Navigation
}

\section{Professor A. Stratton}

F. C. Bell in his contribution raises most interesting philosophical questions. In commenting on them, I will refer to a recent translation (Navigation U.S.A., 14, 26) of Schuler's paper and use the same notation.

Gravity and the Schuler pendulum

The impossibility of distinguishing by any physical measurement between a gravitational force and the inertial reaction force of an accelerating frame of reference is fundamental. In Fig. I our observer in an enclosed laboratory is observing the compression of a linear spring supporting a 'proof mass' $m$ (he knows the natural uncompressed

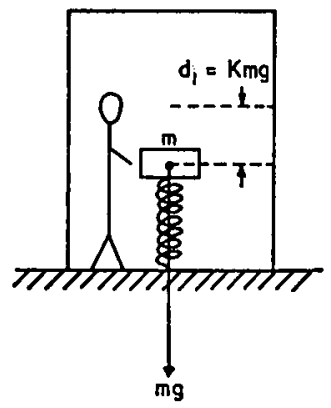

(a)

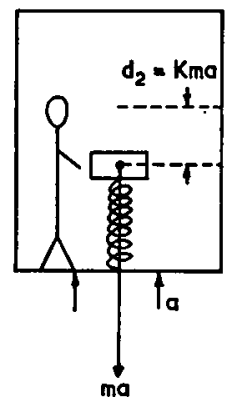

(b)
FIG I (a). Gravitation (b) Inertia length of the spring). In situation I(a) the laboratory-unknown to him-is sitting on the surface of the Earth and the spring is compressed to $d_{1}=k m g$ by the gravitational force of $g$ per unit mass acting on $m$ ( $k$ is a constant of the spring). In situation $\mathrm{I}(\mathrm{b})$ the laboratory-also unknown to himis in free motion in space accelerating under the action of a silent and vibration-free propulsive motor generating a thrust of, $a$, per unit mass of the labora- 
tory and its contents; the spring is compressed to $d_{2}=k m a$ by the inertia of the mass $m$. The compression of the spring will be identical in the two cases if $a=g$ and no observation of the 'proof mass' will distinguish between gravitational force and acceleration of the frame of reference under the (external) propulsive force as the 'source' of the deflection.

Inertial navigation thus requires certain a priori knowledge of the gravitational field; the question for discussion is the nature of the information required.

Consider first of all the compound pendulum of Fig. 2 moving on the surface of a sphere of radius $R, \omega$ being the angular velocity of the radius vector; no gravitational field exists.

The acceleration of the pedulum support $O$ is $R d \omega / d t$ and the inertial reaction force acting at the centre of mass $G$ is $m R d \omega / d t$ ( $m$ is the total mass of the pendulum).

Hence the torque applied to the pendulum is $m R a d \omega / d t$. If $\omega_{1}$ is the angular velocity of the pendulum and $\theta$ the moment of inertia

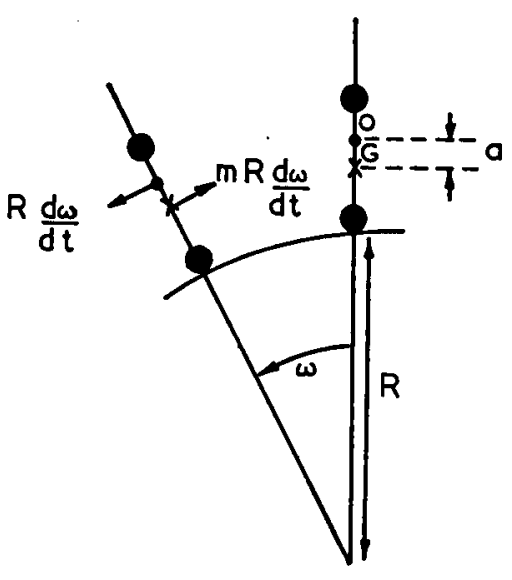

FIG. 2. A compound pendulum

$$
m R a \frac{d \omega}{d t}=\theta \frac{d \omega_{1}}{d t}
$$

If initially the pendulum is aligned to the radius vector then it will remain aligned to the radius vector if $d \omega_{1} / d t=d \omega / d t$, which requires that $R a=\theta / \mathrm{m}$.

If $k$ is the radius of gyration of the pendulum then $\theta=m k^{2}$ and hence $k=\sqrt{ }(R a)$.

If $R=6.37 \times 10^{6}$ metre (equal to the radius of the Earth) and $k=2$ metres then $a=0.6$ micron which, as Schuler remarks, is completely impracticable for a physical pendulum [note: Slater translates ' $4 \mathrm{~m}$ Massenabstand' as 4 metres radius of gyration; this should be (as the above calculation demonstrates) a 'distance between masses of 4 metres'-i.e. a dumbell].

The above derivation is identical with that of Schuler-but there is no gravitational field; the compound pendulum once aligned to the radius vector maintains alignment when moved on the surface of a sphere of radius $R$. If a gravitational field is introduced always parallel to the radius vector then the aligned pendulum being always parallel to the gravitational field is unaffected by it - and no knowledge of the magnitude $g$ of the gravitational field is necessary.

If the pendulum is disturbed, however, it will oscillate with a period

$$
T=2 \pi /\left(\frac{\theta}{m g a}\right)=2 \pi /\left(\frac{k^{2}}{g a}\right)
$$

which is dependent on the magnitude of $g$.

The 'tuning' condition for maintenance of alignment to the radius vector depends, however, only on the radius $R$ of the sphere, viz.

$$
a=\frac{k^{2}}{R}
$$




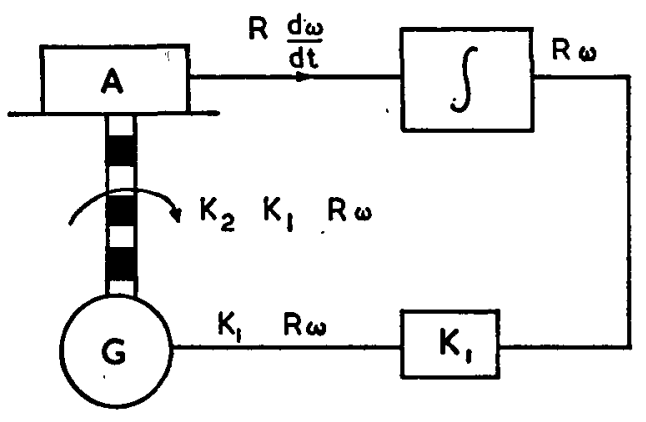

FIG. 3. Accelerometer-integrator-gyroscope combination

Such a pendulum, if it could be physically constructed and dimensioned to maintain alignment to the Earth's radius, when taken to another planet of the same radius but, say, four times the gravitational field, would still maintain alignment to the radius vector but have a period (if disturbed) of only $42 \cdot 2$ minutes.

Now consider the accelerometer - integrator - gyroscope combination referred to by Carr and Scott (Fig. 3) ('inertial platform') operating on a sphere of radius $R$ in the absence of a gravitational field. The output of the accelerometer is $R d \omega / d t$ and the precession signal applied to the gyroscope is $K_{1} R \omega$.

The 'platform' carrying the accelerometer will rotate at a rate

$$
\omega^{1}=K_{1} K_{2} R \omega
$$

If $K_{1} K_{2}=1 / R$ then $\omega^{1}=\omega$ and once aligned normal to the radius vector the platform will, if there are no instrumental errors, stay aligned-even in the absence of a gravitational field.

Instrumental errors-for example an initial velocity error as considered by Bell-will,' in the absence of a gravitational field, cause unbounded divergence of the platform from the radius vector. If there is a gravitational field $g$ parallel to the radius of the sphere then, if disturbed, the platform will oscillate with a period $T=2 \pi \sqrt{ }(R / g)$ and, of great importance, oscillations due to instrumental errors are bounded.

Apart from the practically all important instrumental errors, however, the 'inertial platform' is directly analogous in behaviour to the physically impracticable compound pendulum of Schuler and would also maintain the vertical, without any modification, on a planet of equal radius but

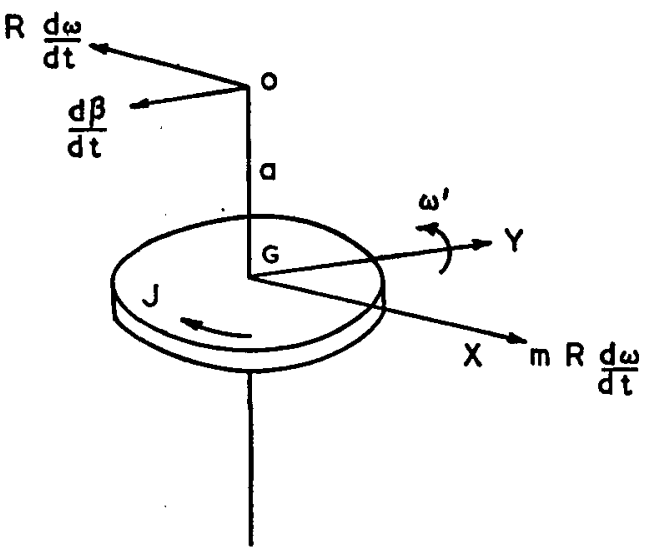

FIG. 4. Platform rotation differing gravitational field.

In contrast, the 'gyroscopic pendulum' of Schuler does not maintain the vertical independent of the gravitational field. Fig. 4 shows such a pendulum moving on the surface of a sphere with acceleration $R d \omega / d t$. The resultant inertial reaction torque $m a R d \omega / d t$ about axis $G Y$ will cause precession at an angular rate $d \beta / d t$ about the orthogonal axis $G X$ where

$$
\frac{J d \beta}{d t}=m a R d \omega
$$


( $J$ being the angular momentum of the wheel.)

Hence if initially aligned to the radius vector the gyroscope will precess about $G X$ through an angle (assumed small) of

$$
\begin{aligned}
\beta & =\frac{m a R \omega}{J} \\
& =\frac{m a v}{J}
\end{aligned}
$$

where $v$ is the velocity over the surface of the sphere. About GY the gyroscope will maintain the initial alignment and will not follow the radius vector with movement over the sphere. If, however, a radial gravitational field $g$ obtains, the tilt induced by equation ( $\mathrm{I}$ ) will generate a gravitational torque mga $\beta$, which in turn will give a precession rate about $G Y$ of

If

$$
\begin{aligned}
\omega^{1} & =\frac{m g a \beta}{J} \\
& =\left(\frac{m^{2} g a^{2} R}{J}\right) \omega
\end{aligned}
$$

i.e.

$$
\frac{m^{2} g a^{2} R}{J^{2}}=1
$$

$$
\frac{m g a}{J}=\sqrt{ }\left(\frac{g}{R}\right)
$$

(Schuler equation I0)

then $\omega^{1}=\omega$ and the gyroscopic pendulum will maintain indication of the vertical in the plane OGX. If translated to a planet with different $g$, but the same $R$, the constants of the instrument would have to be modified.

A gravitational field is thus essential for the operation of the Schuler gyroscopic pendulum.

Summarizing, with the compound pendulum and the inertial platform, it is only necessary to know that the gravitational field is normal to the surface; with the 'gyroscopic pendulum' of Schuler it is also necessary to know the magnitude of the gravitational field.

\section{Schuler and inertial navigation}

Schuler recognized in his paper 'that a gyroscopic horizon with two gyroscopes counter-rotating simultaneously indicates the speed of the vehicle'. He then points out that such an apparatus gives the total velocity of the aircraft relative to Earth and that Earth rotation must be subtracted. He then quite rightly discounts the accuracy of a magnetic compass for determining the direction and inclination of the Earth's axis (which must be known to subtract the Earth's rotation); the only alternative which he offers for this is the stars.

The key to pure inertial navigation-which is omitted by Schuler-is the definition of the Earth's axis by means of a free gyroscope aligned before flight, and with a sufficiently low drift rate to maintain this alignment unmonitored during flight.

It would be very interesting to know the earliest published record of this step. 